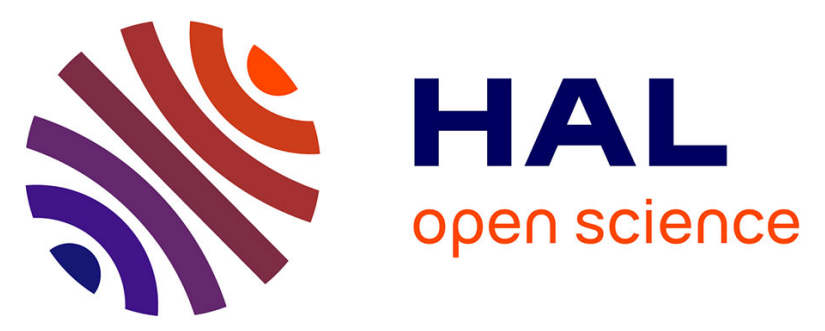

\title{
Simultaneous observations of quasi-periodic ELF/VLF wave emissions and electron precipitation by DEMETER satellite: A case study
}

M Hayosh, D. L. Pasmanik, A. G. Demekhov, Ondřej Santolík, Michel Parrot, E. E. Titova

\section{To cite this version:}

M Hayosh, D. L. Pasmanik, A. G. Demekhov, Ondřej Santolík, Michel Parrot, et al.. Simultaneous observations of quasi-periodic ELF/VLF wave emissions and electron precipitation by DEMETER satellite: A case study. Journal of Geophysical Research Space Physics, 2013, 118, pp.4523-4533. 10.1002/jgra.50179 . insu-01258063

\section{HAL Id: insu-01258063 https://hal-insu.archives-ouvertes.fr/insu-01258063}

Submitted on 18 Jan 2016

HAL is a multi-disciplinary open access archive for the deposit and dissemination of scientific research documents, whether they are published or not. The documents may come from teaching and research institutions in France or abroad, or from public or private research centers.
L'archive ouverte pluridisciplinaire HAL, est destinée au dépôt et à la diffusion de documents scientifiques de niveau recherche, publiés ou non, émanant des établissements d'enseignement et de recherche français ou étrangers, des laboratoires publics ou privés. 


\title{
Simultaneous observations of quasi-periodic ELF/VLF wave emissions and electron precipitation by DEMETER satellite: A case study
}

\author{
M. Hayosh, ${ }^{1}$ D. L. Pasmanik, ${ }^{2}$ A. G. Demekhov, ${ }^{2,3}$ O. Santolik,,${ }^{1,4}$ M. Parrot, ${ }^{5}$ and \\ E. E. Titova ${ }^{6}$ \\ Received 8 February 2012; revised 15 January 2013; accepted 7 February 2013; published 8 July 2013.
}

[1] We present results of case studies of quasi-periodic (QP) ELF/VLF hiss emissions detected onboard the DEMETER satellite. Three events with simultaneous periodic modulation in VLF wave intensity and energetic electron precipitation are found. In each event we observe exact coincidence of one or two busts of VLF wave intensity with energetic electron precipitation peaks. To our knowledge, such observations made onboard satellites have not been reported earlier. All events are observed at fairly quiet geomagnetic conditions $(\mathrm{Kp}<3)$. The dynamic spectrum of the VLF waves in these QP events was characterized by a regular frequency increase in each burst, and the repetition period was less than or about $20 \mathrm{~s}$. These features allow us to suggest that the observed events belong to the QP2-class, i.e., events which are not associated with geomagnetic pulsations. We also analyze energetic electron data from NOAA-17 spacecraft which has helio-synchronous circular orbit similar to DEMETER spacecraft and measured in the same region of the magnetosphere within $30 \mathrm{~min}$ for the analyzed events. NOAA-17 data confirm that the QP emissions were detected by DEMETER in the region of isotropization of energetic electrons, which is typically associated with the development of the cyclotron instability. Modulation of electron flux with a period close to the QP emission period is observed in two events. Based on the observed correlation between bursts of wave intensity and energetic particle flux, we estimate the location and spatial extent of the source region for QP emissions.

Citation: Hayosh, M., D. L. Pasmanik, A. G. Demekhov, O. Santolik, M. Parrot, and E. E. Titova (2013), Simultaneous observations of quasi-periodic ELF/VLF wave emissions and electron precipitation by DEMETER satellite: A case study, J. Geophys. Res. Space Physics, 118, 4523-4533, doi:10.1002/jgra.50179.

\section{Introduction}

[2] Quasi-periodic (QP) VLF emissions are wideband emissions which are observed inside or near the plasmapause [see, e.g., Helliwell, 1965; Sato et al., 1974; Kovner et al. 1977; Tixier and Cornilleau-Wehrlin, 1986; Sazhin and Hayakawa, 1994; Smith et al., 1998]. They are characterized by a periodic modulation of the wave intensity with typical periods from several seconds up to a few minutes. Generation of QP emissions is usually accompanied by pre-

\footnotetext{
${ }^{1}$ Institute of Atmospheric Physics, Prague, Czech Republic.

${ }^{2}$ Institute of Applied Physics RAS, Novgorod, Russia.

${ }^{3}$ Department of Space Physics, Lobachevsky State University of Nizhny Novgorod, Novgorod, Russia.

${ }^{4}$ Faculty of Mathematics and Physics, Charles University in Prague, Prague, Czech Republic.

${ }^{5}$ LPC2E/CNRS, Orléans, France.

${ }^{6}$ Polar Geophysical Institute of the Kola Science Centre RAS, Apatity, Russia.

Corresponding author: M. Hayosh, Department of Space Physics, Institute of Atmospheric Physics, Bočni II, 1401, 14131 Prague 4, Czech Republic. (hayosh@ufa.cas.cz)

(C)2013. American Geophysical Union. All Rights Reserved. 2169-9380/13/10.1002/jgra.50179
}

cipitation of energetic electrons, which is also modulated with the same period [see, e.g., Smith et al., 1998].

[3] Sato et al. [1974] suggested to divide QP emissions into two classes according to their relation with geomagnetic activity: QP emissions which are closely associated with geomagnetic pulsations of the same period (QP1 class) and those which do not correlate with geomagnetic pulsations (QP2 class). Later, Sato and Fukunishi [1981] categorized QP emissions on the basis of their spectral forms. They divided QP emissions into five types based on frequencytime spectra and suggested a phenomenological model for explanation of different types of the frequency-time spectra of QP emissions.

[4] An idea for theoretical explanation of the modulation mechanism for QP2 emissions was proposed by Bespalov and Trakhtengerts [1976] and Davidson [1979]. It was based on a relaxation oscillation regime of wave generation which may take place in the presence of a constant source of energetic electrons with transverse anisotropic distribution function. Further studies showed [Bespalov, 1981] that under certain conditions, the relaxation oscillations can turn into self-sustaining oscillations. Similar results were obtained by Davidson [1986] on the basis of a phenomenological model. 


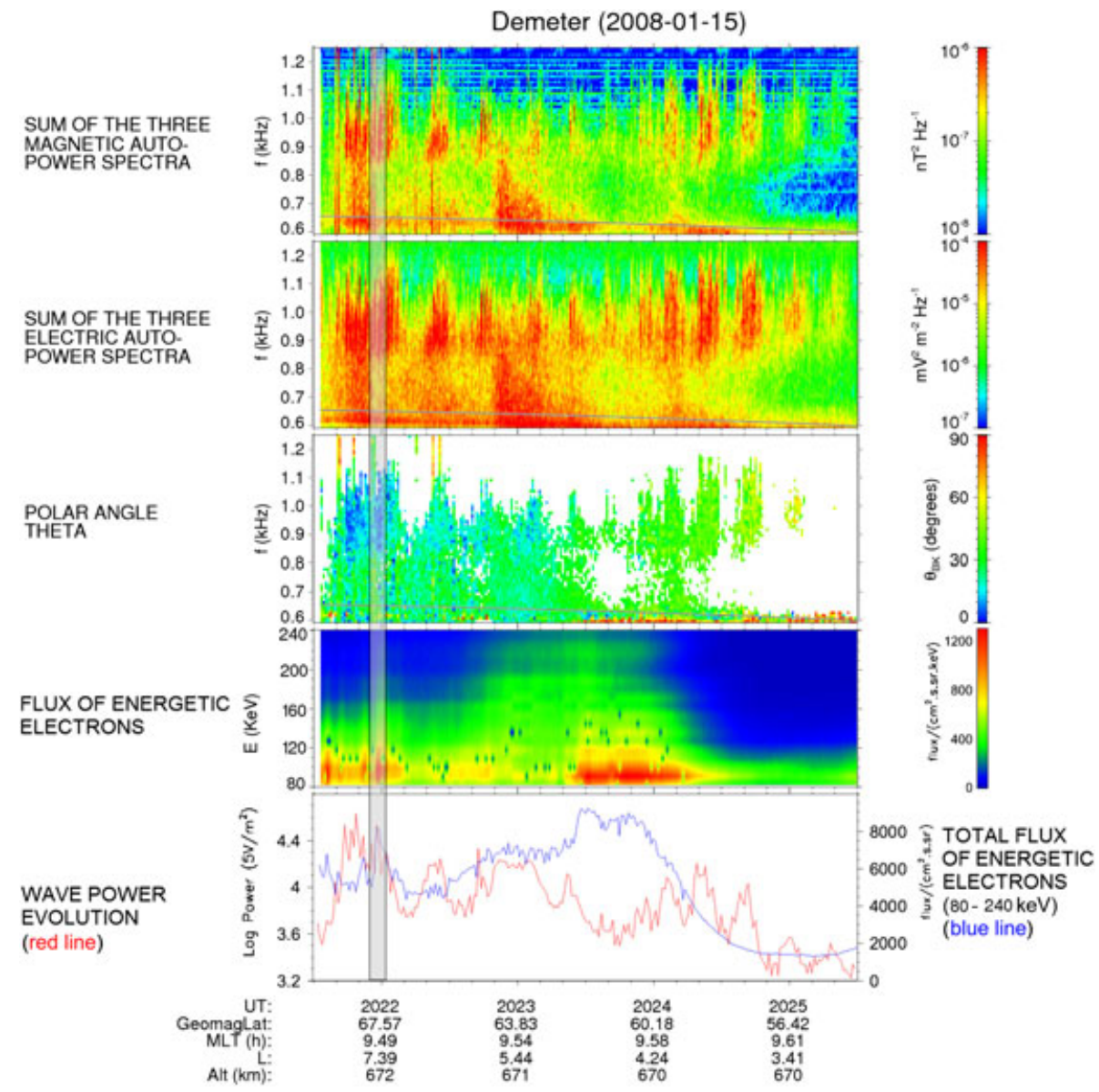

Figure 1. Electromagnetic field and energetic electron measurements onboard DEMETER satellite during burst operation mode in the orbit 18910 0 on February 11, 2008, between 2021 UT and 2026 UT: magnetic (first panel) and electric (second panel) field power spectra and polar angle of wave vector (third panel) for VLF emissions; energetic electron flux (fourth panel); wave power (red line) in the frequency band of observed QP emissions and total flux of energetic electrons (blue line) in the energy interval 80-240 keV (fifth panel). Time interval corresponding to simultaneous observation of wave power burst and peak in energetic electron flux is marked by gray bar.

[5] Trakhtengerts et al. [1986] and Demekhov and Trakhtengerts [1994] developed a more rigorous kinetic model of the flow cyclotron maser (FCM) to explain pulsating aurorae patches. This model allows us to describe different periodic regimes of wave generation with different shapes of the dynamic spectrum. Further study of this model was performed by Pasmanik et al. [2004a, 2004b], where the dependence of possible generation regimes on the parameters of the energetic-electron source was analyzed and these results were applied to case studies of QP emissions observed by Freja and Magion 5 spacecraft. A good correspondence between observations and theoretical simulations for selected QP events was demonstrated. A method for theoretical evaluation of missing magnetospheric plasma parameters by use of available data and theoretical modeling was suggested. To our knowledge, there are currently no reports on direct simultaneous observations of QP emissions and accompanying energetic electron precipitation and analysis of their correlation.

[6] Rocket [Sandahl et al., 1980] and satellite [Sato et al., 2004] measurements revealed a good correlation between spatial and temporal variations of the precipitating electron flux and the optical pulsating aurora. Simultaneous groundbased observations of VLF waves and pulsating aurorae by Hansen et al. [1988]; Zaitsev et al. [1994], Novikov et al. [1994], and Tagirov et al. [1999] demonstrated good correspondence between the VLF wave intensification and the auroral patches in several events. Analysis of delay time between observation of VLF wave bursts and corresponding optical pulsation performed by Hansen et al. [1988] demonstrated that source region for waves and precipitating particles is located in the near-equatorial region. Spatiotemporal variation of fast-moving auroral patches and their connection with generation of structured VLF emissions was reported by Novikov et al. [1994]. Tagirov et al. [1999] revealed correlation between intensifications of the VLF waves and optical pulsation in a particular auroral patch.

[7] Nishimura et al. [2010] studied the correlation between whistler-mode waves observed onboard THEMIS spacecraft and pulsating aurora observed by a ground-based all-sky imager. They have found an almost one-to-one correspondence of the auroral pulsations with whistler wave burst and calculated the correlation coefficients for VLF waves intensity and auroral intensity for different auroral patches. 


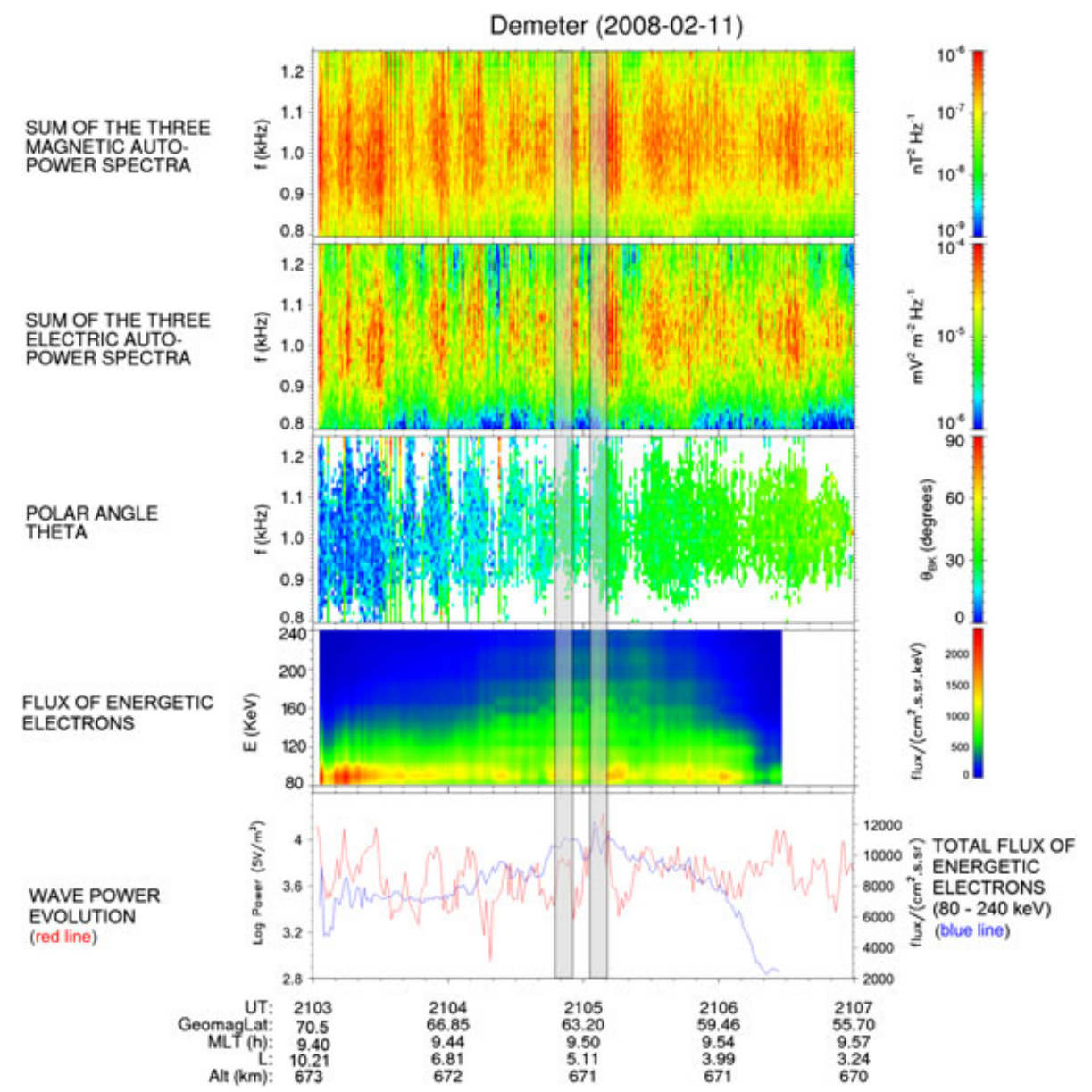

Figure 2. Same as on Figure 1 for QP event on 11 February 2008.

Based on these results, the magnetic field line with footprint at the auroral patch with highest correlation coefficient was identified as the source for observed VLF emissions.

[8] In this paper, we report results of case studies of QP emissions observed by the DEMETER satellite. Several QP events observed during burst operation mode of the satellite were selected. The most interesting feature of these events is simultaneous observation of periodic modulation in both wave intensity and energetic electron precipitation. Energetic particle data from the DEMETER satellite are supplemented by data from the NOAA-17 satellite whose trajectory is very similar to the DEMETER trajectory, and on this basis properties of the wave-generation region are discussed.

\section{Instrumentation and Observations}

[9] We use wave and particle data from the French microsatellite DEMETER [Parrot et al., 2006] for our investigation of the VLF QP emissions. The DEMETER spacecraft was launched in June 2004 on a quasi helio-synchronous circular orbit (10.30-22.30 LT) with a $98^{\circ}$ inclination and an altitude of about $700 \mathrm{~km}$ and operated till December 2010. It performed 14 orbits per day and provided measurements from $-65^{\circ}$ to $65^{\circ}$ geomagnetic latitudes.
[10] We analyzed the wave magnetic field data from the IMSC instrument [Parrot et al., 2006], and the wave electric field data from the ICE device [Berthelier et al., 2006] measured in the ELF/VLF bands (from $15 \mathrm{~Hz}$ to $17.4 \mathrm{kHz}$ ). These measurements were made in the survey or burst modes of operations of the DEMETER instruments. The survey mode was used to record low bit rate data along the entire trajectory of the spacecraft around the Earth, compared to the burst mode with much higher data resolution, which was turned on only above regions of the greatest interest. In the analyzed cases, the burst mode was typically switched on at latitudes near the poleward edges of the measurement regions.

[11] Three components of both electric and magnetic fields were measured in the frequency range from $15 \mathrm{~Hz}$ to $1.25 \mathrm{kHz}$ in the burst mode. These waveform data allow us to perform the wave analysis, namely to compute the k-vector and polarization of the detected waves. We have used for this analysis the method of Singular Value Decomposition (SVD) [Santolik et al., 2003, 2006a]. In the upper frequency band (up to $17.4 \mathrm{kHz}$ ), power spectra of the electric and magnetic field components were computed onboard with a frequency resolution of $19.5 \mathrm{~Hz}$ and a time resolution of $2 \mathrm{~s}$ in both operation modes.

[12] High-energy particle flux data were obtained from measurements of the IDP instrument [Sauvaud et al., 2006]. 


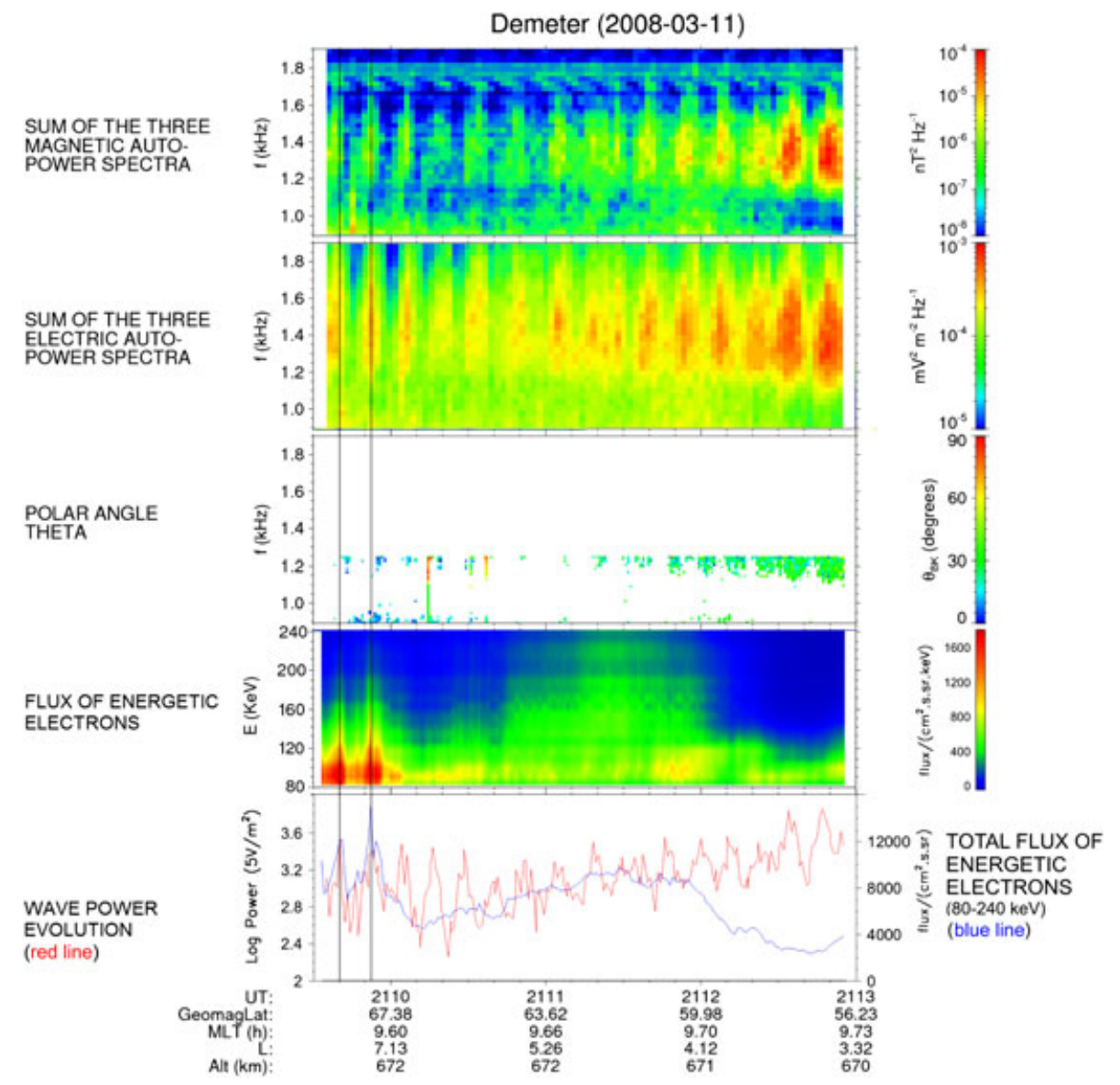

Figure 3. Same as on Figure 1 for QP event on 11 March 2008. Correlation between wave power bursts and peaks in energetic electron flux is marked by vertical lines.

This detector measured electron fluxes in the energy range from $72.9 \mathrm{keV}$ up to $2.5 \mathrm{MeV}$ divided into 256 energy channels. Time resolution of measurements in the survey and in the burst modes were 7 and $1 \mathrm{~s}$, respectively. The detector measured particles with pitch angles close to $90^{\circ}$ with respect to the geomagnetic field with an aperture $\pm 16^{\circ}$, i.e., trapped particles. However, due to the low altitude of the DEMETER satellite (about $680 \mathrm{~km}$ ), these particles are actually very close to the equatorial loss cone boundary: their equatorial pitch angle is about $1-2^{\circ}$ larger than the loss cone angle (loss cone boundary varies from $\sim 8^{\circ}$ for $L=3$ to $\sim 1.5^{\circ}$ for $L=8$ ).

[13] Analysis of the DEMETER satellite data reveals more than 100 events with QP emissions seen during 3 years of observations (2006-2008) in the ELF/VLF frequency band.

[14] In our study we supposed that QP emissions propagate almost along the geomagnetic field lines. This direction of the wave propagation can be verified by the calculation of the wave vector. We are able to calculate the wave vector only for frequencies lower than $1.2 \mathrm{kHz}$ where electric and magnetic field components are measured during burst operations of the DEMETER instruments. We describe the wave vector by the polar and azimuth angles. We examined first the polar angle showing the deviation of the wave vector from the local magnetic field line at the observation point. On the other hand, for our investigation we need simultane- ous observations of both the QP emissions and the energetic particles flux which have similar period of fluctuations at the same time. Taking into account these two criteria we have chosen only three events of the QP emissions observations from the total number of events. As one can see, the discussed quasi-periodic wave and particle burst were observed only in a small number of cases. Possible reasons for that are discussed below.

[15] Event of 15 January 2008. Figure 1 shows DEMETER data for VLF waves and energetic electron flux during burst operation mode in the orbit 18910_0 on 15 January 2008, between 2021 UT and 2026 UT. The first to third panels show magnetic and electric field power spectra and polar angle of the wave vector for VLF emissions. The evolution of energetic electron flux in the energy interval from 81.8 to $242 \mathrm{keV}$ is shown on the fourth panel. Wave power evolution (red line) in the frequency band from 0.6 to $1.2 \mathrm{kHz}$ and total flux of energetic electrons (blue line) in the energy interval from 80 to $240 \mathrm{keV}$ are shown in the fifth panel.

[16] The DEMETER passed the morning sector (MLT changes from 0940 to 0965) in the Northern Hemisphere. The satellite moved from the auroral region to low latitudes at the geomagnetic latitudes from $69^{\circ}$ to $54^{\circ}$ (corresponding to $L$ from 8.75 to 3 ). The geomagnetic activity for this event was low with $\mathrm{Kp}=2-$ to 2 and Dst varying between -20 and $-10 \mathrm{nT}$ during the day. However, the AE index has shown a disturbed period of $4 \mathrm{~h}$ which started $8 \mathrm{~h}$ before the event. 


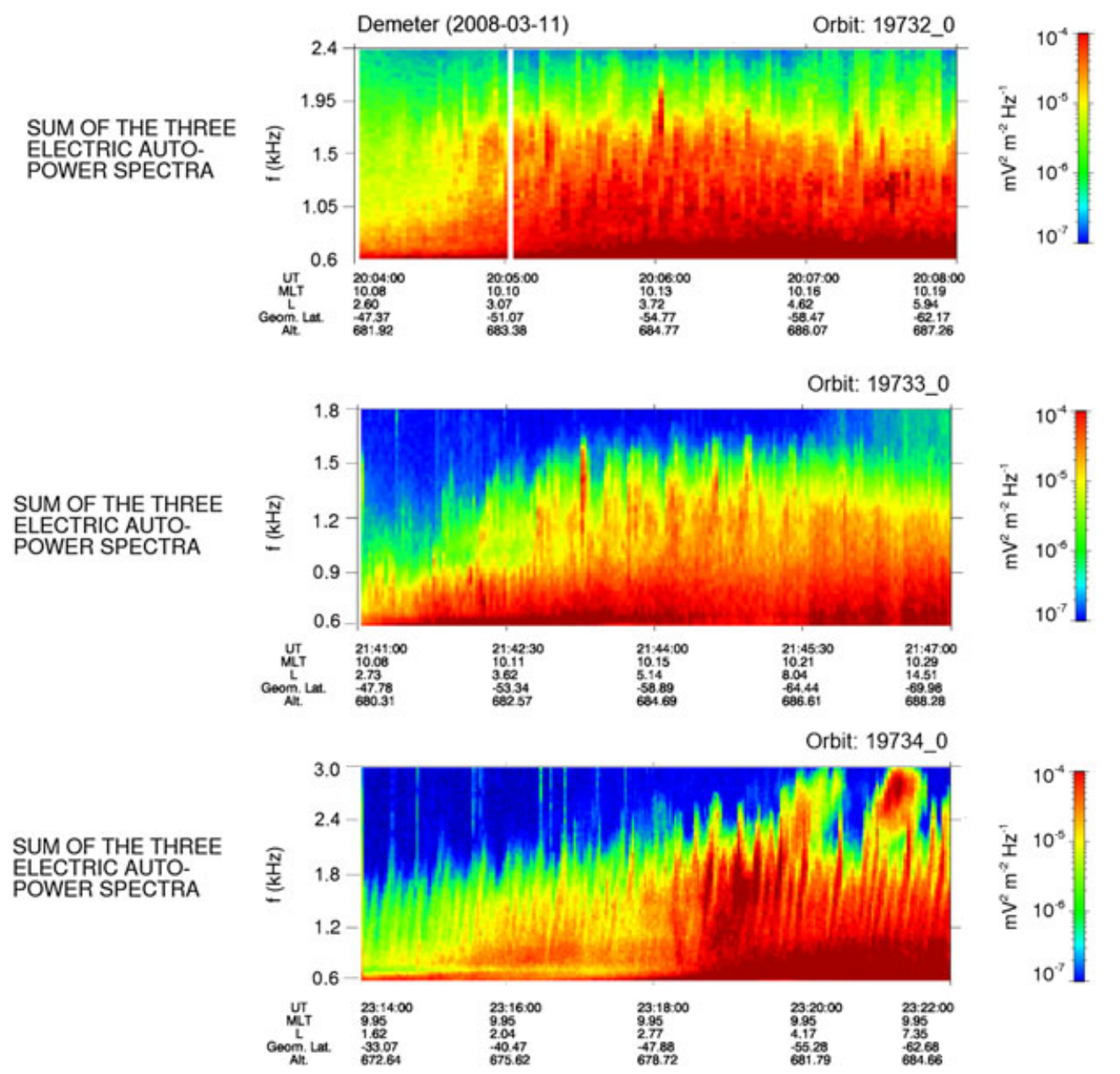

Figure 4. Electric field spectra of VLF emissions on sequential orbits for event on 11 March 2008.

This period of increase of the AE values up to about $500 \mathrm{nT}$ indicates sub-storm activity.

[17] As is seen from Figure 1, the frequency range of QP emissions is approximately from 850 to $1200 \mathrm{~Hz}$, and their period is about $18 \mathrm{~s}$. QP emissions were observed in a wide range of geomagnetic latitudes. The spectrum of individual QP bursts preserved the same shape with diffuse structure during the entire event. Weakly pronounced frequency
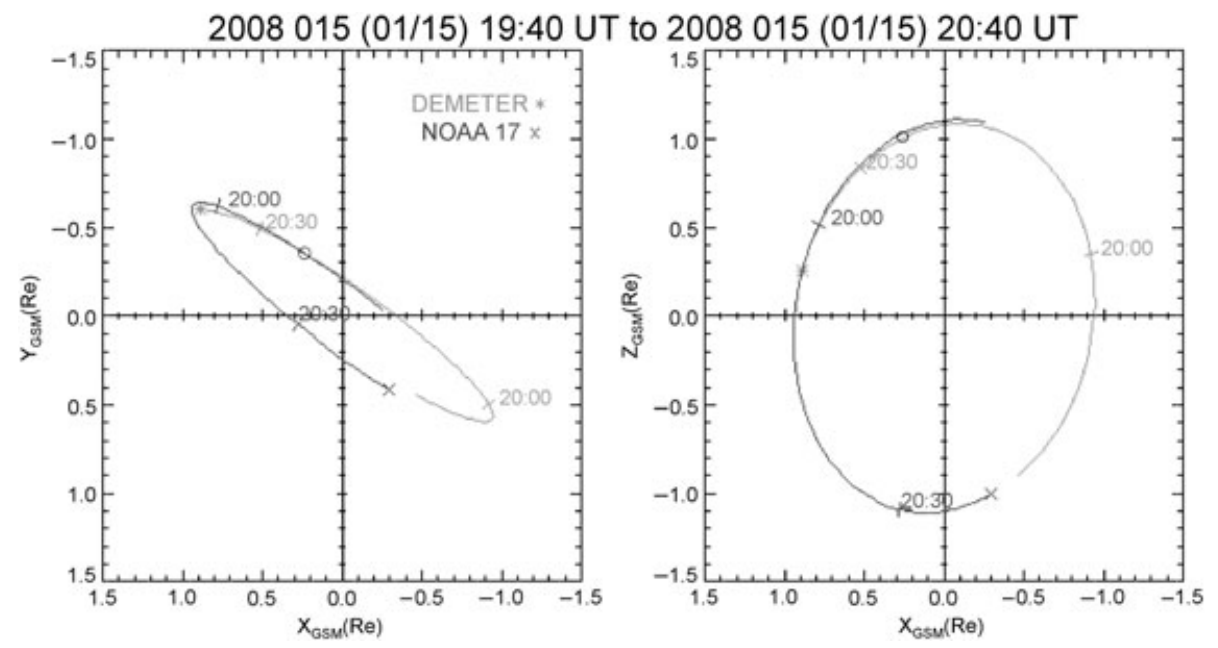

Figure 5. Trajectories of DEMETER (light gray) and NOAA-17 (dark gray) satellites in the geomagnetic coordinates on 15 January 2008, UT from 1940 to 2040. The DEMETER position corresponding to observation of electron precipitation simultaneous with QP emissions (about 2022 UT) is marked by a circle. 

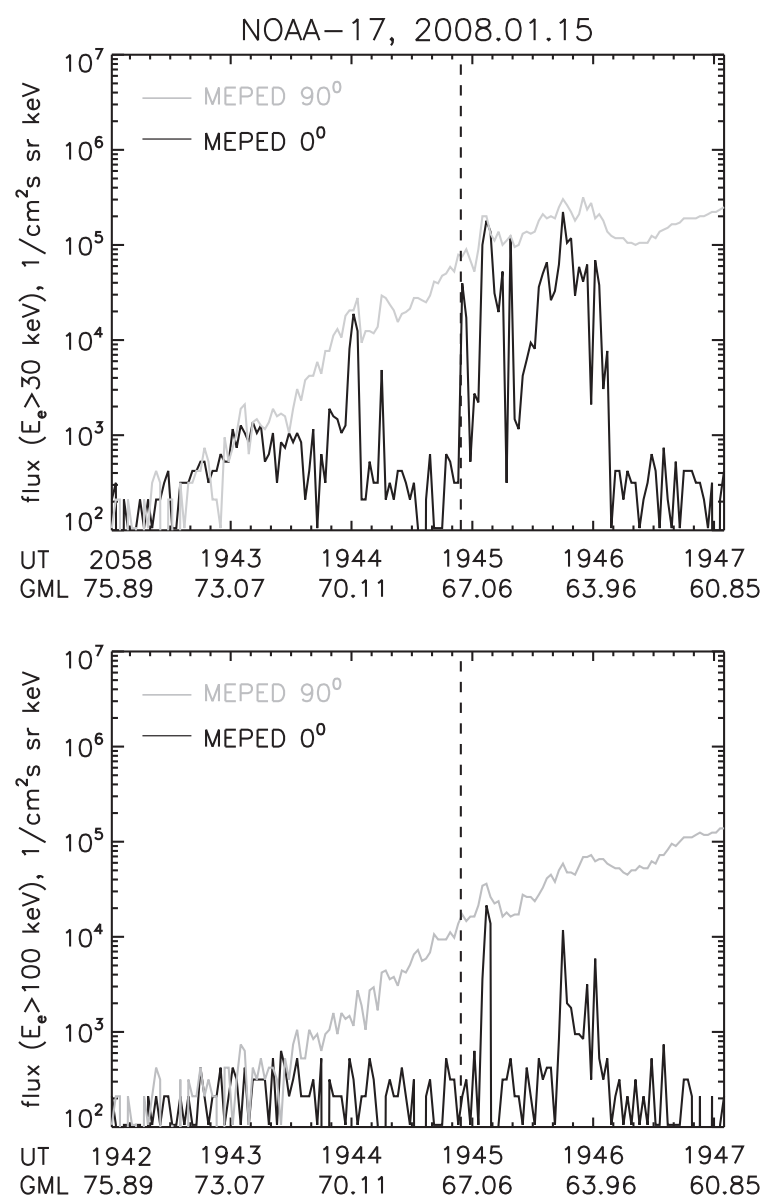

Figure 6. Energetic particles data from NOAA-17 satellite for QP event on 15 January 2008. Data from two energy channels $E>30 \mathrm{keV}$ (upper panel) and $E>100 \mathrm{keV}$ (lower panel) and for pitch-angles $0^{\circ}$ (black line) and $90^{\circ}$ (gray line) is shown. Geomagnetic latitude at which synchronous bursts of waves and particles were observed by DEMETER is marked by a dashed line.

rise for VLF emission bursts with value of approximately $15-20 \mathrm{~Hz} / \mathrm{s}$ can be seen. Such spectrum is similar to typical observations of QP2 events on the ground [Sato and Fukunishi, 1981].

[18] The polar angle of wave vector (third panel) is less than $30^{\circ}$, i.e., the QP emissions propagate at a small angle to the geomagnetic field. Using the six components of the electromagnetic field, it is also possible to calculate the wave polarization. This calculation shows that the ellipticity and the planarity of polarization are both very close to 1 , i.e., the waves in this QP event are circularly polarized.

[19] Further analysis of the full wave vector direction (not shown) completes the results by additional information that the waves propagate from the magnetosphere toward the Earth and that their wave vectors are slightly inclined toward the equator in the plane of the local magnetic meridian.

[20] An increase in the flux of energetic electrons in the lower energy part of the electron detector range during this event is seen (fourth panel). The flux intensity is modulated at the beginning of the event.
[21] One may notice that the burst of wave power and the burst of energetic electron flux occur very close to each other in time (almost simultaneously at about 2022 UT, the time interval is marked by a gray bar). This behavior of the waves and particles suggests that the bursts might be linked.

[22] Analysis of DEMETER data on adjacent orbits revealed QP emissions with similar diffuse structure of wave bursts on a preceding orbit at about $1850 \mathrm{UT}$, i.e., $1.5 \mathrm{~h}$ before the QP emissions from Figure 1. These emissions had a similar repetition period of about $13 \mathrm{~s}$, but were in a higher frequency band from 2 to $3.5 \mathrm{kHz}$. DEMETER operated at lower latitudes only on this orbit, and QP emissions were observed at latitudes from $50^{\circ}$ to $40^{\circ}$ ( $L$ from $\sim 3.7$ to $\sim 2.2$ ). It is therefore reasonable to assume that these QP emissions originated from a different source than the QP emissions presented in Figure 1.

[23] Event of 11 February 2008. Figure 2 shows DEMETER data for the burst operation mode in the orbit 193070 on 11 February 2008, between 2103 UT and 2107 UT. The geomagnetic activity was fairly low with $\mathrm{Kp}=2-3$ and Dst varying between -30 and $-20 \mathrm{nT}$, and $\mathrm{AE}$ index actively changes during at least $12 \mathrm{~h}$ before the event, with clear local maximum of $500 \mathrm{nT}$ occurring approximately four hours before the event. The same data format as in Figure 1 is chosen; wave power at the fifth panel was calculated for the frequency band from 0.8 to $1.2 \mathrm{kHz}$.
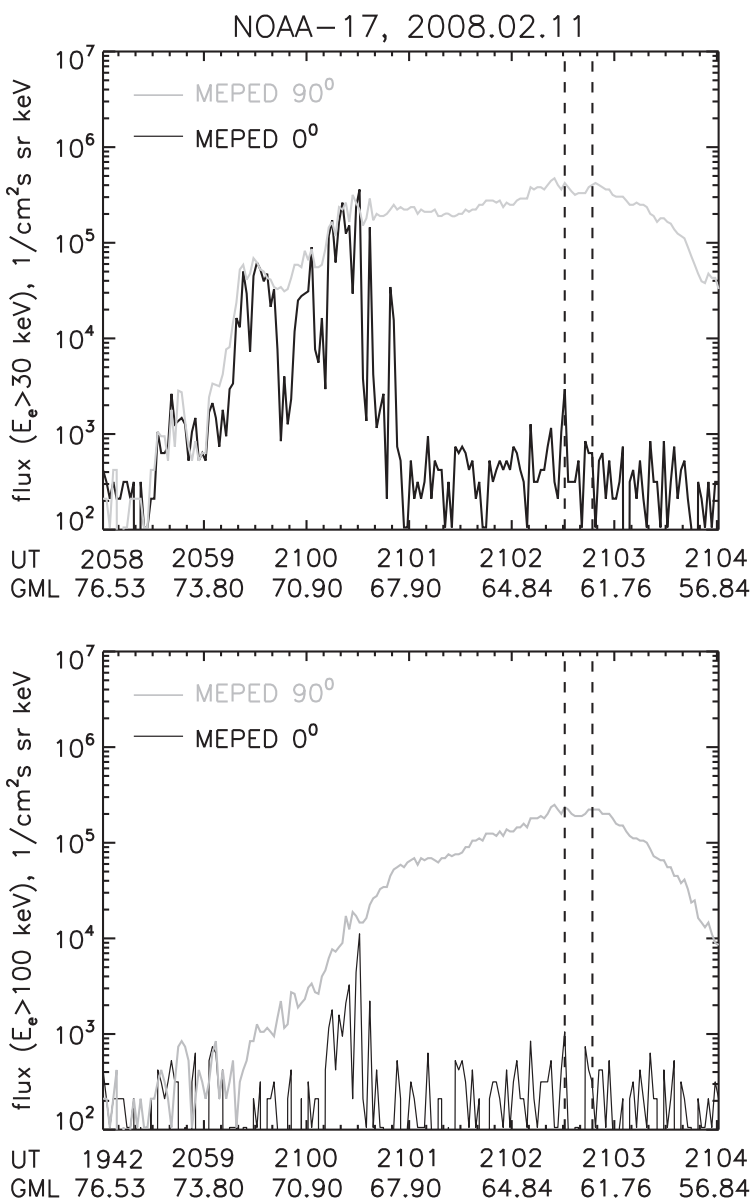

Figure 7. Same as on Figure 7 for QP event on 11 February 2008. 

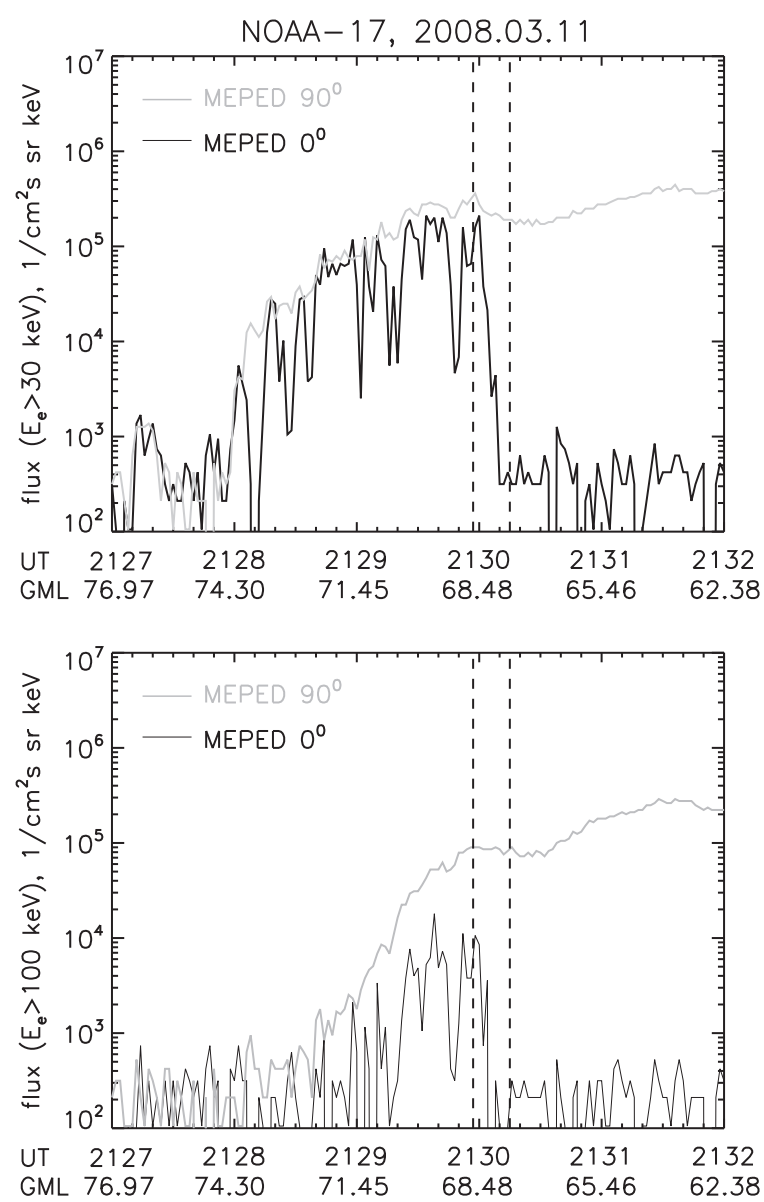

Figure 8. Same as on Figure 7 for QP event on 11 March 2008.

[24] Similar to the event of 15 January 2008, DEMETER was in the morning sector (MLT changes from 0940 to 0957) in the Northern Hemisphere and moved toward lower latitudes with geomagnetic latitude varying from about $70^{\circ}$ to $55^{\circ}$ ( $L$ values from 10.2 to 3.2 ).

[25] The QP emissions were observed in the frequency range from about 850 to $1250 \mathrm{~Hz}$ and their period is about $16 \mathrm{~s}$. The emission bursts are of a similar shape as in previous events. The frequency rise inside a single burst can be estimated as $40-50 \mathrm{~Hz} / \mathrm{s}$. The waves propagate with a small angle of less than 30 degrees to the geomagnetic field (Figure 1, third panel).

[26] Modulation of the energetic electron flux is seen at the beginning of the event (fourth panel). It shows a slow increase with time and has maxima with two relatively diffused peaks at about 2105. At the same time, two bursts of VLF emissions power are observed at about 2104:52 and 2105:08 and this modulation can be associated with the particle peaks. However, the presence of neighboring peaks of lower amplitude, together with unclear boundaries of the peaks, does not allow us to make the definite conclusion about possible correlation between waves and particle in this case.

[27] The QP emissions with a similar spectral structure were detected also in the Southern Hemisphere on the southern part of the same orbit at about 2135 UT, i.e., half an hour after QP emissions presented in Figure 2. The properties of the QP emission were very similar. The repetition period is about $15 \mathrm{~s}$, the frequency band is from 0.7 to $1.5 \mathrm{kHz}$, and the emissions were observed at a wide range of latitudes: from $-50^{\circ}$ to $-68^{\circ}$ ( $L$ from $\sim 3$ to $\sim 12$ ). DEMETER operated in the survey mode on this part of the orbit. Based on these data, we can expect that these QP emissions have the same origin as those observed in the Northern Hemisphere (Figure 2).

[28] Event of 11 March 2008. The third selected event with QP emissions was observed in the orbit 19733 0 on 11 March 2008, between 2109 UT and 2113 UT. The geomagnetic activity was very low with $\mathrm{Kp}=1+-2$ and Dst varying between -25 and $-5 \mathrm{nT}$. In this case AE index had a disturbed period with a local peak of about $700 \mathrm{nT}$ occurring 6 $\mathrm{h}$ before the event, another small peak occurring $5 \mathrm{~h}$ before the event, and a subsequent decay down to about $100 \mathrm{nT}$ by the time of the event.

[29] The DEMETER data for this event are shown in Figure 3. Wave power in the frequency range of $1.0-1.8 \mathrm{kHz}$ is shown on the bottom panel.

[30] Similar to both events described above, DEMETER was in the morning sector (MLT changes from 0935 to 0940) in the Northern Hemisphere and moved toward lower latitudes with geomagnetic latitude varying from $70^{\circ}$ to $55^{\circ}$ ( $L$ values from 8.5 to 3.5 ).

[31] The third selected event with QP emissionsThe QP emissions were observed in the frequency range from 1100 to $1600 \mathrm{~Hz}$, the period is about $14 \mathrm{~s}$, and the rise of tones for VLF emission bursts is more pronounced in this event at about $50 \mathrm{~Hz} / \mathrm{s}$. On the whole, the emission bursts are similar in shape to the events described above.

[32] The third selected event with QP emissionsAs was noted in the previous Section, six components of the electromagnetic field are measured only for frequencies below $1250 \mathrm{kHz}$. Thus the wave polar angle can be estimated only for the lower frequency part of the QP emission bursts. However, it is seen that the polar angle is small (less than $20^{\circ}$ ) in this frequency range.

[33] The third selected event with QP emissionsA rather strong increase in energetic electron flux (fourth panel) occurs at the beginning of this event. Both peaks in electron flux at 2109:40 and 2109:54 (marked by vertical lines) have good correlation with the bursts of VLF emissions.

[34] The third selected event with QP emissionsOn this day, QP emissions were observed by DEMETER during a very long time interval from 1934 to 0030 UT, at both Northern and Southern Hemispheres, during three sequential morning-side orbits (19732_0, 19733_0 and 19734_0). Figure 4 presents VLF measurements of the electric field for each of these sequential orbits. The properties of the QP emissions observed at about 2144 UT (half of hour after event in Figure 3) at the southern part of orbit 19733_0 were very similar to those presented in Figure 3 . The repetition period is about $16 \mathrm{~s}$, frequency band is from 1 to $1.6 \mathrm{kHz}$, and the latitude changes from $-50^{\circ}$ to $-64^{\circ}$ ( $L$ from $\sim 3.5$ to $\sim 11)$. Similarly to the previously discussed events, we can expect that these QP emissions had the same origin as those observed in the northern part of the orbit (Figure 3). The QP emissions of both adjacent orbits had a shorter period of about $10 \mathrm{~s}$ and a frequency band from 0.8 to $3 \mathrm{kHz}$. Those 
emissions could also originate from the same source which if its properties are changing in time.

\section{Discussion}

[35] As one can see, all three selected events with QP emissions presented in the previous section exhibit rather similar features:

[36] 1. the observed QP emissions were observed by DEMETER in the morning sector at $L$-shells 4-10;

[37] 2. the QP emissions have the same spectral structure with similar frequency ranges, frequency rise rates inside a single burst, and close periods of about 15-20 s. These properties do not change much during each event;

[38] 3. the QP emissions are formed by VLF waves propagating at a small angle to the geomagnetic field (less than $40^{\circ}$, with nearly circular wave polarization;

[39] 4. all events have been observed under rather quiet geomagnetic conditions. However, the perturbations of the AE index indicate sub-storms which always occurred 4-6 h before the events;

[40] 5. an increase in energetic particle flux at energies about $100 \mathrm{keV}$ takes place in all events, and a coincidence of one or two VLF wave bursts with peaks in energetic electron flux was observed.

[41] Results of the wave analysis (angle of propagation and wave polarization) allow us to assume that the waves were detected near the same magnetic flux tube as their source is located, and this source is related to cyclotron interactions with energetic electrons. QP emissions with rising-tone elements detected during low magnetic activity usually belong to the QP2 class emissions, and the morning sector is a typical region for observation of QP2 emissions [Sato and Fukunishi, 1981]. Moreover, periods of observed QP emissions do not match the period of any typical geomagnetic pulsations (flux-tube oscillations), which usually belong to the Pc3-5 ranges for subauroral and auroral latitudes. On this basis, we suggest that the presented events belong to the QP2 class. The repetition periods of the VLF waves $(\sim 15-20 \mathrm{~s})$ are on the order of the modulation period of pulsating aurora and, therefore, could be associated with auroral pulsations [e.g., Johnstone, 1983; Trakhtengerts et al., 1986], however, this association cannot be verified from the available data.

[42] Since the QP emissions preserve the same spectral structure during the event, it is reasonable to assume that all wave pulses in one event originate from the same source. The wide spatial extent of the observation zone in geomagnetic latitudes and $L$-shells can be explained by the spreading of the VLF wave energy during the propagation from the source which is located in the equatorial region. According to the results of Jiriček et al. [1981], Santolik et al. [2006b], and Bortnik et al. [2011], the $L$-value of a ray originated from the equatorial source could change by several units. On the contrary, if we assume that the observed waves originate from sources located at different $L$-shells, then we should expect a strong variation in the frequency band of the VLF waves, which depends on local (equatorial) gyrofrequency, and the latter varies by more then an order of magnitude in each event (for $L$ varying from 3 to 9).

[43] An interesting feature of the observations presented in this paper is the simultaneous observation of VLF waves and precipitating electrons (strictly speaking, those near the equatorial loss cone) and the correlation in their modulation. To our knowledge, such correlated observations made onboard satellites have not been reported earlier.

[44] Note that the electron-precipitation bursts synchronous with VLF wave pulses were observed in much narrower intervals of latitudes than the wave bursts in each event. The fact that this correlation was seen only for one or two successive bursts of QP emissions has an obvious explanation. Energetic electrons almost do not shift across the magnetic field on a time scale of their motion from the equator to the ionosphere. Therefore, energetic electrons which participate in the generation of QP emissions and, hence, precipitate into the ionosphere can be detected only at the footprint of the generation region. The source of QP emissions is usually associated with particular geomagnetic flux tube which has a rather small cross-section. On the contrary the VLF waves can illuminate much wider area (up to several $L$-shells on the low altitudes), since their propagation is not strictly field-aligned.

[45] On this basis, we can suggest that observation of increased particle fluxes with peaks correlating with bursts of VLF waves can indicate the flux tube in which the source of QP emissions is located.

[46] Let us note that these features of wave and particle propagation impose rather strong restrictions for simultaneous observation of the related wave and particle bursts by a low-orbiting satellite, since it requires that the satellite should pass the footprint of the source region.

[47] We note that, in the event on 11 February 2008, synchronous bursts of waves and particles are seen at two points. However, the latitude is rather high at the beginning of this event (Glat $\approx 70^{\circ}, L \approx 10$ ). Cyclotron waveparticle interactions, which are believed to be the cause of QP emission, are efficient only at frequencies below half of the equatorial electron gyrofrequency. Thus, QP emissions in the frequency range from 0.8 to $1.2 \mathrm{kHz}$ could not be generated at this latitude (the equatorial gyrofrequency at Glat $=70$ is less than $1 \mathrm{kHz}$ ). It seems more reasonable to assume that the source is located at lower latitudes (Glat $\approx 63^{\circ}$, $L \approx 5$ ) where synchronous bursts are seen the second time during this event. It is also possible that in this case two separate source regions existed, but we cannot determine both of them based on DEMETER data.

[48] Based on the theoretical approach for QP generation mechanism developed by Pasmanik et al. [2004a, 2004b] we can estimate properties of the plasma in the source region (i.e., in the near-equatorial region) from the cyclotron resonance condition at the equator:

$$
\omega_{\min } \simeq \omega_{H} / \beta_{*}, \quad \beta_{*}=n_{c} W_{0}\left(\frac{8 \pi e^{2} m}{c^{2} \omega_{H}^{2}}\right)
$$

where $\omega_{\min }$ is the lowest frequency of QP emissions, $\omega_{H}$ is the equatorial gyrofrequency, $n_{c}$ is the equatorial cold 
plasma density, $W_{0}$ is the characteristic energy of energetic electrons, $e$ and $m$ are the electron charge and mass, and $c$ is the velocity of light in free space.

[49] Substituting the values from the first event on 15 January $2008\left(\omega_{\min } \sim 0.85 \mathrm{kHz}, L \sim 7.4\right.$ and $\left.W_{0} \sim 100 \mathrm{keV}\right)$ into this equation, we obtain $n_{c} \approx 1 \mathrm{~cm}^{-3}$. This is a reasonable value for the corresponding geomagnetic latitudes. A similar value $\left(n_{c} \approx 0.8 \mathrm{~cm}^{-3}\right)$ is obtained from this estimation for the third event on 11 March 2008. For the second event on 11 February 2008, we have $\omega_{\min } \sim 0.85 \mathrm{kHz}, L \sim 5$ and $W_{0} \sim 100 \mathrm{keV}$, which gives $n_{c} \approx 100 \mathrm{~cm}^{-3}$.

[50] Propagation directions of the observed QP emissions in Figures 1-3 consistently show a pattern of increasing wave-normal angles as the spacecraft moves from the subauroral region toward the equator. This observation is consistent with previous experimental results on propagation directions of low altitude hiss [Santolik and Parrot, 1998; Santolik et al., 2006b]. Ray tracing simulations of these waves [Chum and Santolik, 2005; Santolik et al., 2006b] indicate that their source, if it is located in the equatorial plane, generates waves at very oblique wave-normal angles (close to the Gendrin angle) inclined toward the Earth. This seems to contradict with the assumptions used in equation (1), where a simplified approach for quasi-parallel propagating waves is used. Equation (1) and the plasma density estimates which follow from it must be therefore understood as an order-of-magnitude estimate for which this simplified approach is still useful. On the other hand, the ray tracing simulations, in general, also have to use rather strong assumptions on the density profiles along the ray paths. The wave-normal directions obtained by this theoretical calculation cannot be taken for granted unless they are confirmed by direct measurements.

[51] The correlation between wave and precipitating particle bursts can also be used to estimate the cross-section of the magnetic flux tube of the generation region at the altitude of DEMETER. Bearing in mind that satellite horizontal velocity is about $7.6 \mathrm{~km} / \mathrm{s}$, we can obtain a size of the generation region of less than $110 \mathrm{~km}$ for the first event, for which the wave-particle correlation is observed in a single burst. For two other events, for which a correlation for two bursts is observed, the generation region is less than $140 \mathrm{~km}$ (estimated as the distance passed by the satellite between the detection of those bursts). Mapping along this magnetic flux tube to the equatorial region gives an estimate of the source region size of about $3000 \mathrm{~km}$.

[52] It is seen from the energetic particle spectra measured by DEMETER (see fourth panel in Figures 1-3) that an increase in electron flux occurs for the electrons near the low-energy cutoff of the particle detector. Unfortunately the lowest energy observed by DEMETER instrument is $72.9 \mathrm{keV}$; whereas particles with lower energies should also be involved in the generation of QP emissions.

[53] To supplement the DEMETER data for energetic electrons, we searched for available data from other satellites. We found that the NOAA-17 satellite operated at the same time and had a trajectory very similar to the DEMETER trajectory. This is illustrated in Figure 5 for the event of 15 January 2008 . Both satellite paths are shown in geomagnetic coordinates in the $X_{\mathrm{GSM}}-Z_{\mathrm{GSM}}$ ) and $X_{\mathrm{GSM}}-Y_{\mathrm{GSM}}$ planes for the time interval from 1940 to 2040 UT. The DEMETER position corresponding to the observation of electron pre- cipitation together with QP emissions (at about 2022 UT) is marked by a circle. The NOAA-17 satellite was at the same location at about 1945 UT, i.e., 35 min earlier.

[54] Energetic particle measurements by the MEPED instrument onboard the NOAA-17 satellite [Raben et al., $1995]$ in the energy channels $E>30 \mathrm{keV}$ (upper panel) and $E>100 \mathrm{keV}$ (lower panel) for pitch angles corresponding to precipitation electrons (black line marked as $0^{\circ}$ ) and corresponding to trapped electrons (gray line marked as $90^{\circ}$ ) are shown in Figure 6. We note that NOAA MEPED detectors have $\pm 15^{\circ}$ field of view and $0^{\circ}$ detector is oriented close the Earth radial direction. For latitudes corresponding to discussed events, we can roughly say that the $0^{\circ}$ detector measures particles inside the loss cone (whose boundary is about $50^{\circ}$ at the altitude of the NOAA satellite), and $90^{\circ}$ detector measured electrons outside the loss cone. This accuracy is sufficient for the purpose of our paper. The time resolution of the MEPED instrument is $2 \mathrm{~s}$. A strong increase in the trapped $\left(\Theta=90^{\circ}\right.$, black line) particle flux is seen in both channels as the satellite moves trough the radiation belt (UT from 1943 to 1954). A drastic increase in precipitating $\left(\Theta=0^{\circ}\right.$, gray line) particle flux up to the same order of magnitude as the trapped flux is observed in both energy channels near 1945 UT. Thus, the region of energetic particle isotropization is observed at practically the same location where QP emissions were detected. Distinct peaks in precipitating particle flux and a simultaneous increase in trapped flux are seen at about 1945 UT in the energy channel $E>30 \mathrm{keV}$. The time interval between peaks is $10-30 \mathrm{~s}$ which is close to the period of QP emissions observed by the DEMETER satellite. The peaks can also be seen in the higher energy channel (Figure 6, bottom panel). The geomagnetic latitude at which synchronous bursts of waves and particles were observed by DEMETER is marked by a dashed line. We can see that it coincides almost exactly with the peak seen in the NOAA-17 data.

[55] The generation of QP emissions can last from several minutes up to tens of minutes. Thus, we can expect that the isotropization observed by the NOAA-17 satellite could also be due to the generation of QP emissions belonging to the same event which was detected by DEMETER 35 min later. Unfortunately, the available data do not allow us to confidently relate the NOAA-17 and DEMETER observations as belonging to the same event. Nevertheless, based on the fact that QP emissions were detected by DEMETER even earlier on the previous morning-side orbit, it is reasonable to assume that QP emissions observed on both DEMETER orbits and precipitating particle modulation observed by NOAA-17 35 min before the second DEMETER flyby belong to the same QP event.

[56] Measurements of energetic particles onboard NOAA17 for the second event on 11 February 2008 are shown on Figure 7 . In this case the satellites were very close to each other. The NOAA-17 satellite was about 3 min ahead of DEMETER. QP emissions were observed by DEMETER from about 2103 UT, and NOAA-17 passed the same region at about $2100 \mathrm{UT}$. Increase in the precipitating particle flux in both channels $(E>30 \mathrm{keV}$ and $E>100 \mathrm{keV})$ is seen in this case at 2059-2101 UT. This location corresponds to the beginning of measurements by DEMETER. No apparent modulation in the electron flux is seen during this time interval. Taking into account the above mentioned peculiarities 
of the efficiency of cyclotron interactions, this isotropization could not be associated with QP emissions presented in Figure 2.

[57] Similar to Figure 6, geomagnetic latitudes corresponding to synchronous bursts of waves and precipitating electrons in the DEMETER data are marked by vertical dashed lines in Figure 7. A rather weak isotropization of the energetic electron distribution can be explained by a weak diffusion regime of cyclotron interactions in the source region.

[58] Figure 8 shows NOAA-17 measurements for the third selected event of 11 March 2008. In this case, the NOAA-17 satellite was about 20 min later than DEMETER: QP emissions were observed by DEMETER from about 2109 UT, and NOAA-17 entered the same region at about 2129 UT. An increase in the trapped particle fluxes in both channels $(E>30 \mathrm{keV}$ and $E>100 \mathrm{keV})$ is also seen in this case at 2128-2130 UT.

[59] Modulation of electron flux of the precipitating particles is seen in both energy channels between 2129 and 2130 UT. The time interval between peaks is about 12-16 s which is very close to the period of QP emissions observed by DEMETER. Similar to above, dashed vertical lines mark the location at which the correlation between waves and electron bursts is seen in the DEMETER data. The difference in latitudes of the observations of peaks in the precipitating electron flux by DEMETER and NOAA-17 may be related to the slightly different paths of the satellites across magnetic flux tube associates with the source region (orbits spacing was about $80 \mathrm{~km}$ ). This also could be related to the temporal modification of the source region.

\section{Conclusions}

[60] In this paper we present results of case studies of the correlation between VLF QP emissions observed by the DEMETER satellite and energetic electron precipitation detected by the same spacecraft. To our knowledge, it is the first reported observations of such a direct correlation.

[61] Based on geomagnetic conditions and the properties of the observed QP-emissions (their spectral structure, frequency drift, and repetition period), we can identify them as belonging to the QP2 class, i.e., the temporal modulation is not caused by geomagnetic pulsations.

[62] It is shown that exact coincidence of one or two bursts of VLF wave intensity with energetic electron precipitation peaks takes place. This allows us to identify the location of the magnetic flux tube at which the generation of the observed QP emissions occurs and to estimating the size of the generation region as about $100-150 \mathrm{~km}$ along the DEMETER trajectory.

[63] The spatial and temporal extents of the observed energetic electron precipitation events correlated with the QP emissions are also analyzed on the basis of NOAA-17 satellite measurements of energetic-electron flux. These data confirm that QP emissions were observed inside the region of energetic electron izotropization, i.e., in the region with favorable conditions for the development of the cyclotron instability. This is consistent with the theoretical model by Pasmanik et al. [2004a], explaining generation of such QP emissions based on the development of the cyclotron instability in a magnetospheric duct or region with enhanced cold plasma density. Modulation of electron flux with the same period as the QP emission has been also observed.

[64] Acknowledgments. This work is based on observations performed by the DEMETER satellite launched by the Centre National d'Etudes Spatiales. The authors thank J.J. Berthelier, the PI of the ICE instrument, for the use of the data. NOAA MEPED data were provided by NOAA ftp://poes.ngdc.noaa.gov/sem/poes/data/full/. Work at Institute of Atmospheric Physics Prague was supported by the P209-11-2280 grant of the Grant Agency of the Czech Republic and LH12231. DP, AD, and ET were partially supported by the Russian Foundation for Basic Research (project 11-02-00654-a and 11-02-00397-a) and by the Russian Academy of Sciences (Basic Research Program 22).

\section{References}

Berthelier, J. J., et al. (2006), ICE, the electric field experiment on DEMETER, Planet. Space Sci., 54, 456-471, doi:10.1016/j.pss.2005. 10.016.

Bespalov, P. A. (1981), Self-modulation of radiation of a plasma cyclotron maser, JETP Lett., 33(4), 182-185.

Bespalov, P. A., and V. Y. Trakhtengerts (1976), The dynamics of the cyclotron instability in a magnetic trap, Fiz. Plazmy, 2(3), 397-406.

Bortnik, J., L. Chen, W. Li, R. M. Thorne, and R. B. Horne (2011), Modeling the evolution of chorus waves into plasmaspheric hiss, J. Geophys. Res., 116(A15), A08221, doi:10.1029/2011JA016499.

Chum, J., and O. Santolík (2005), Propagation of whistler-mode chorus to low altitudes: Divergent ray trajectories and ground accessibility, Ann. Geophys., 23(12), 3727-3738, doi:10.5194/angeo-23-3727-2005.

Davidson, G. T. (1979), Self-modulated VLF wave-electron interactions in the magnetosphere: A cause of auroral pulsations, J. Geophys. Res., 84 (A11), 6517-6523.

Davidson, G. T. (1986), Pitch angle diffusion in morningside aurorae 2. The formation of repetitive auroral pulsations, J. Geophys. Res., 91, 4429-4436.

Demekhov, A. G., and V. Y. Trakhtengerts (1994), A mechanism of formation of pulsating aurorae, J. Geophys. Res., 99(4), 5831-5841.

Hansen, H. J., E. Mravlag, and M. W. J. Scourfield (1988), Coupled 3and $1.3-\mathrm{Hz}$ components in auroral pulsations, J. Geophys. Res., 931, 10,029-10,034, doi:10.1029/JA093iA09p10029.

Helliwell, R. A. (1965), Whistlers and Related Ionospheric Phenomena, Stanford University Press, Palo Alto, California.

Jiřiček, F., V. E. Jurov, E. E. Titova, and P. Tř́ska (1981), Localisation of VLF emissions observed in the plasma-trough region, Adv. Space Res., 1, 369-372, doi:10.1016/0273-1177(81)90136-8.

Johnstone, A. D. (1983), The mechanism of pulsating aurora, Ann. Geophys., 1, 397-410.

Kovner, M. S., V. A. Kuznetsova, and I. I. Likhter (1977), On the midlatitude modulation of fluxes of energetic electrons and VLF emissions, Geomagn. Aeron. (English Transl), 17, 867-870.

Nishimura, Y., et al. (2010), Identifying the driver of pulsating aurora, Science, 330, 81-84, doi:10.1126/science.1193186.

Novikov, Y. P., A. A. Mironov, E. E. Titova, T. A. Yahnina, V. R. Tagirov, S. A. Chernouss, V. I. Salin, J. Manninen, and T. Turunen (1994), Spatialtemporal variations of pulsating auroral patches and their relationship with VLF emissions of chorus type, Geomagn. Aeron., 5, 32-36.

Parrot, M., et al. (2006), The magnetic field experiment IMSC and its data processing onboard DEMETER: Scientific objectives, description and first results, Planet. Space Sci., 54, 441-455, doi: $10.1016 /$ j.pss.2005.10.015

Pasmanik, D. L., A. G. Demekhov, V. Y. Trakhtengerts, and M. Parrot (2004a), Modeling whistler wave generation regimes in magnetospheric cyclotron maser, Ann. Geophys., 22, 3561-3570, doi:10.5194/angeo-223561-2004.

Pasmanik, D. L., A. G. Demekhov, V. Y. Trakhtengerts, and M. Parrot (2004b), Quasi-periodic ELF/VLF wave emissions in the Earth's magnetosphere: Comparison of satellite observations and modeling, Ann. Geophys., 22, 4351-4361, doi:10.5194/angeo-22-4351-2004.

Raben, V. J., D. S. Evans, H. H. Sauer, S. R. Sahm, and M. Huynh, (1995), TIROS/NOAA satellite Space Environment Monitor data archive documentation: 1995 update, NOAA Tech. Memo., ERL SEL-86, NOAA, Boulder, Colo., http://docs.lib.noaa.gov/rescue/TIROS/ QC8075U6S3no86.pdf.

Sandahl, I., L. Eliasson, and R. Lundin (1980), Rocket observations of precipitating electrons over a pulsating aurora, Geophys. Res. Lett., 7, 309-312, doi:10.1029/GL007i005p00309.

Santolík, O., and M. Parrot (1998), Propagation analysis of electromagnetic waves between the helium and proton gyrofrequencies in 


\section{HAYOSH ET AL.: QUASI-PERIODIC EMISSIONS OBSERVED BY DEMETER SATELLITE}

the low-altitude auroral zone, J. Geophys. Res., 103, 20,469-20,480, doi:10.1029/98JA01386.

Santolík, O., M. Parrot, and F. Lefeuvre (2003), Singular value decomposition methods for wave propagation analysis, Radio Sci., 38(1), 1010, doi:10.1029/2000RS002523.

Santolík, O., F. Němec, M. Parrot, D. Lagoutte, L. Madrias, and J. J. Berthelier (2006a), Analysis methods for multi-component wave measurements on board the DEMETER spacecraft, Planet. Space Sci., 54, 512-527, doi:10.1016/j.pss.2005.10.020.

Santolík, O., J. Chum, M. Parrot, D. A. Gurnett, J. S. Pickett, and N. Cornilleau-Wehrlin (2006b), Propagation of whistler mode chorus to low altitudes: Spacecraft observations of structured ELF hiss, J. Geophys. Res. Space Phys., 111(A10), A10208, doi:10.1029/2005JA011462.

Sato, N., and H. Fukunishi (1981), Interaction between ELF-VLF emissions and magnetic pulsations: Classificaton of quasi-periodic ELF-VLF emissions based on frequency-time spectra, J. Geophys. Res., 86(A1), 1929.

Sato, N., K. Hayashi, S. Kokubun, T. Oguti, and H. Fukunishi (1974), Relationship between quasi-periodic VLF-emission and geomagnetic pulsation, J. Atmos. Terr. Phys., 36(9), 1515-1526.

Sato, N., D. M. Wright, C. W. Carlson, Y. Ebihara, M. Sato, T. Saemundsson, S. E. Milan, and M. Lester (2004), Generation region of pulsating aurora obtained simultaneously by the FAST satellite and a Syowa-Iceland conjugate pair of observatories, J. Geophys. Res., 109 (A18), A10,201, doi:10.1029/2004JA010419.
Sauvaud, J. A., et al. (2006), High-energy electron detection onboard DEMETER: The IDP spectrometer, description and first results on the inner belt, Planet. Space Sci., 54, 502-511, doi:10.1016/j.pss.2005.10.019.

Sazhin, S. S., and M. Hayakawa (1994), Periodic and quasiperiodic VLF emissions, J. Atmos. Terr. Phys., 56(6), 735-753.

Smith, A. J., M. J. Engebretson, E. M. Klatt, U. S. Inan, R. L. Arnoldy, and H. Fukunishi (1998), Periodic and quasiperiodic ELF/VLF emissions observed by an array of Antarctic stations, J. Geophys. Res., 103, 23,61123,622.

Tagirov, V. R., V. S. Ismagilov, E. E. Titova, V. A. Arinin, A. M. Perlikov, J. Manninen, T. Turunen, and K. Kaila (1999), Auroral pulsations and accompanying VLF emissions, Ann. Geophys., 17, 66-78, doi:10.1007/s00585-999-0066-9.

Tixier, M., and N. Cornilleau-Wehrlin (1986), How are the VLF quasiperiodic emissions controlled by harmonics of field line oscillations? The results of a comparison between ground and GEOS satellites measurements, J. Geophys. Res., 91, 6899-6919.

Trakhtengerts, V. Y., V. R. Tagirov, and S. A. Chernous (1986), A circulating cyclotron maser and impulsive VLF emissions, Geomagn. Aeron. 26(1), 77-82.

Zaitsev, D. B., Y. P. Novikov, V. R. Tagirov, and S. A. Chernouss (1994), Diagnostics of spatial-temporal parameters of auroral ionosphere on the basis of optical and low-frequency electromagnetic data, Geomagn. Aeron., 5, 27-31. 